\title{
Fatores que interferem no prazer e na atividade sexual em mulheres
}

\author{
Factors that interfere with women's pleasure and sexual activity
}

Factores que interfieren en el placer y la actividad sexual de las mujeres

Nathalia Jung Ferreira Malta ${ }^{1 *}$, Maria Elisa Wotzasek Cestari ${ }^{1}$, Rosely Jung Pisicchio ${ }^{1}$, Keli Regiane Tomeleri da Fonseca Pinto, Giovana Garbelini de Souza1, Fabiana Fontana Medeiros².

\section{RESUMO}

Objetivo: analisar os fatores que interferem no prazer e na atividade sexual em mulheres em idade fértil. Método: estudo transversal analítico, realizado em uma unidade de Atenção Primária à Saúde do Sul do Brasil, durante a consulta de enfermagem para realização de exame Papanicolau de 215 mulheres, por meio de instrumento semiestruturado para transcrição de dados do prontuário. Para análise dos dados utilizou-se Teste Qui-quadrado $(p \leq 0,05)$. Resultados: houve significância estatística a utilização de métodos contraceptivos, sendo que o mais utilizado foi o método hormonal, atividade sexual $(p=0,003)$ e prazer $(p=0,012)$, seguida do método cirúrgico, atividade sexual $(p=0,016)$; método de barreira, atividade sexual $(p=0,020)$ e prazer $(p=0,029)$. A dispareunia e sangramento apresentou associação à atividade sexual $(p=0,013)$ e prazer sexual $(p=<0,001)$. Conclusão: considerando a complexidade da mulher perante as relações de amor e erotismo, conclui-se que a saúde reprodutiva da mulher deve ser valorizada e assistida de forma holística, favorecendo o empoderamento das mulheres.

Palavras-chave: Comportamento sexual, Saúde sexual, Saúde pública.

\begin{abstract}
Objective: to analyze factors that interfere with pleasure and sexual activity in women of childbearing age. Method: an analytical cross-sectional study carried out in a Primary Care Unit for Health in Southern Brazil, during the nursing visit to perform a Pap smear of 215 women, using a semi-structured instrument for transcribing data from the medical record. Chi-square test $(p \leq 0.05)$ was used for data analysis. Results: the use of contraceptive methods was statistically significant, and the most used was the hormonal method, sexual activity $(p=0.003)$ and pleasure $(p=0.012)$, followed by the surgical method, sexual activity $(p=0.016)$; barrier method, sexual activity $(p=0.020)$ and pleasure $(p=0.029)$. Dyspareunia and bleeding showed association with sexual activity $(p=0.013)$ and sexual pleasure $(p=0.001)$. Conclusion: considering the complexity of women in relation to love and erotic relationships, it is concluded that women's reproductive health should be valued and assisted in a holistic way, favoring the empowerment of women. oils, fish, oilseeds, teas and spices. Conclusion: It is concluded that the active adult population participating in this study has inadequate knowledge about functional foods, which are not included in their usual diet.
\end{abstract}

Key words: Sexual behavior, Sexual health, Public health.

\section{RESUMEN}

Objetivo: analizar los factores que interfieren con el placer y la actividad sexual en mujeres en edad fértil. Método: estudio transversal analítico, realizado en una unidad de Atención Primaria a la Salud del Sur de Brasil, durante la consulta de enfermería para realización de examen Papanicolaou de 215 mujeres, por medio de instrumento semiestructurado para la transcripción de datos del prontuario. Para el análisis de los datos se utilizó la prueba Chi-cuadrado $(p \leq 0,05)$. Resultados: hubo significancia estadística la utilización de métodos anticonceptivos, siendo que el más utilizado fue el método hormonal, actividad sexual $(p=0,003)$ y placer $(p=0,012)$, seguida del método quirúrgico, actividad sexual $(p=0,016)$; método de barrera, actividad sexual $(p=0,020)$ y placer $(p=0,029)$. La dispareunia y sangrado presentó asociación a la actividad sexual

\footnotetext{
${ }^{1}$ Universidade Estadual de Londrina (UEL), Londrina-PR. *E-mail: nathalia_jferreira@hotmail.com

2 Doutoranda da Universidade Estadual de Londrina (UEL), Londrina- PR.
} 
$(p=0,013)$ y placer sexual $(p=<0,001)$. Conclusión: considerando la complejidad de la mujer ante las relaciones de amor y erotismo, se concluye que la salud reproductiva de la mujer debe ser valorada y asistida de forma holística, favoreciendo el empoderamiento de las mujeres.

Palabras clave: Comportamiento sexual, Salud sexual, Salud pública.

\section{INTRODUÇÃO}

A mulher deve ser responsável e ter o direito de controlar e decidir livremente sobre questões relacionadas à sua sexualidade. Isso inclui a saúde sexual e reprodutiva livre de coação, discriminação e ou violência. Ela deve almejar um relacionamento igualitário no que se refere à relação sexual e a reprodução (MINISTERIO DA SAÚDE, 2013).

O relacionamento saudável e equilibrado deve estar pautado em atitudes e ações com caráter de complementaridade e de horizontalidade, com funções bem distribuídas entre os parceiros, bem como compartilhamento de tarefas e das responsabilidades do casal (LEVY L e GOMES IC, 2008). O desenvolvimento sexual equilibrado não deve basear-se em uma hierarquização sexual, mas sim em uma relação em que cada indivíduo mantenha suas particularidades, autonomia e diferenças (SOUZA NS, et al., 2016).

Nos tempos antigos o homem era o que tinha maior alfabetização e prioridade, excluindo as mulheres, determinando a elas a responsabilidade da criação dos filhos, cuidados domésticos e ainda, a obrigação de suprir o homem em seus desejos (BARBOSA HHG e ALMEIDA JUNIOR VA, 2017). Esse processo foi descrito por Beauvoir, enfatizando que a mulher desde cedo investiu nos valores sexuais que detém, procurando agradar o seu parceiro (BEAUVOIR SO, 1980).

O processo para que a mulher se empodere e conquiste sua autonomia na sociedade, demandou e ainda demanda um grande esforço de reconstrução da sua dignidade social, sobretudo a integridade do seu corpo, não só pelo processo de discriminação da igualdade, mas, também, por meio da violação corporal de distintos níveis, podendo chegar à violência física (BARBOSA HHG e ALMEIDA JUNIOR VA, 2017).

Muitas mulheres permanecem em um relacionamento no qual negam sua própria satisfação para atender aos desejos do parceiro, e mantém o relacionamento afetivo em detrimento do alcance da sua plenitude sexual. O pensamento romântico e ingênuo das relações, principalmente nas mulheres mais jovens, persiste e revela a imaturidade nas relações em que o primordial não é o desenvolvimento sexual saudável (KOBAYASHI C e REIS AS, 2015).

Atualmente, as mulheres têm se preocupado com alterações que ocorrem em seu corpo tais como mudanças hormonais, psicológicas, corporais e o envelhecimento, inclusive com relação ao prazer sexual, extraindo o foco do ato sexual somente para a reprodução (ALVES et al, 2014). Essa nova atitude revela o amor e o erotismo no desenvolvimento sexual feminino, colocando o corpo da mulher de forma ativa na experimentação da sua sexualidade (SOUZA NS, et al., 2016).

O amor, definido pelos psicanalistas pela procura ou desejo do indivíduo de receber ou dar prazer ou ainda; por proporcionar satisfação à outra pessoa, é uma necessidade sexual instintiva de receber e oferecer proteção (SCHLOSSER A e CAMARGO BV, 2014). No entanto a pornografia possui característica de venda, de lucro e o objetivo de proporcionar excitação sexual para aqueles que a consome. A pornografia diferenciase do chamado erotismo, o qual pode estar associado a uma escolha de caráter mais refinado, com ar de beleza e encanto nas abordagens relacionadas ao sexo (MENEZES LO e LIMA FAM, 2015).

Considerando a hipótese de que a mulher moderna apresenta um novo perfil de comportamento em relação a sua sexualidade, incluindo amor e erotismo nas suas relações, e que se faz imprescindível as ações de saúde inovadoras para garantir os direitos da mulher e de sua autonomia durante a relação sexual, o presente estudo teve o objetivo de analisar os fatores que interferem no prazer e na atividade sexual em mulheres. 


\section{MÉTODOS}

Trata-se de um estudo transversal analítico, com abordagem quantitativa. O estudo foi realizado em uma Unidade Básica de Saúde (UBS) em um município situado na região sul do Estado do Paraná.

O município pesquisado possui uma população estimada de 96.733 mil habitantes, com $51,1 \%$ de mulheres, com estimativa de $32,4 \%$ destas mulheres se encontrarem em idade fértil, com idade entre 10 e 49 anos. A população do território da unidade de atenção à saúde na qual o estudo foi realizado é composta por 6.541 mil habitantes, com 51,2\% mulheres, destas 32,7\% possuem idade entre 10 e 49 anos (IBGE, 2015).

A população do estudo foi composta por 215 mulheres atendidas em uma UBS no período de janeiro de 2014 a dezembro de 2016, e que realizaram consulta de enfermagem para prevenção do câncer ginecológico (coleta de material para exame de Papanicolau e exame clínico das mamas). Foram incluídas na pesquisa mulheres que participavam das consultas realizadas pelos graduandos de enfermagem de uma universidade estadual do norte do Paraná, durante estágio curricular.

O Registro para a coleta de dados ocorreu no período de março a julho de 2018, utilizando instrumento semiestruturado para transcrição de dados do prontuário com informações referente a anamnese e exame ginecológico. O registro de dados foi realizado por alunos de graduação de enfermagem. Os dados de mulheres já participantes nos anos anteriores ao estudo foram excluídos, bem como, das mulheres que não responderam às variáveis dependentes: atividade sexual e prazer sexual.

O instrumento para a coleta de dados teve como variável independente: dados socioeconômicos e demográficos, tais como: idade, profissão, estado civil, índice de massa corpórea (IMC), atividade sexual, repouso, trabalho doméstico, dores musculares, número de gestações, tipos de parto e abortos, métodos contraceptivos, queixas sexuais, satisfação com o corpo e motivo da insatisfação e como variável dependente: atividade sexual e prazer sexual na relação.

Os dados foram tabulados por meio do Microsoft Office-Excel® e analisados no programa Statistical Package for the Social Scienc (SPSS) e versão 20.0, considerando os resultados estatisticamente significantes, utilizou-se o Teste Qui-quadrado $(p \leq 0,05)$ e por meio de valores absolutos e medidas de frequência relativa. As variáveis dependentes foram avaliadas por meio do teste de regressão binária logística, para avaliação da razão de chances (RC) e intervalo de confiança (IC) de 95\% (IC 95\%).

A variável queixa sexual foi recategorizada e excluída a categoria outras queixas e, o método contraceptivo foi agrupado em quatro categorias: barreira, hormonal, cirúrgico e não utiliza, sendo descartado dispositivo intrauterino (DIU) e método natural devido a não utilização do método na população do estudo.

Essa pesquisa faz parte de um projeto maior, intitulado: Perfil das mulheres que realizaram exame de Papanicolaou, em unidade de saúde de um munícipio do estado do Paraná. Aprovado pelo Comitê de Ética e Pesquisa (CEP) da Universidade Estadual de Londrina (UEL), com número CAAE 65211816.3.0000.5231. Obteve ainda autorização na Secretaria do Município da Saúde do munícipio, respeitando os princípios da Resolução 466/2012 do Ministério da Saúde.

\section{RESULTADOS}

A população do estudo foi composta por 215 mulheres, com média de idade de 41,38 (Desvio padrão (DP) $=14,08)$ anos, $56,6 \%$ (123) possuíam uma ocupação, 72\% (155) eram casadas ou tinham união estável, $34,4 \%(73)$ tinham ensino fundamental incompleto.

Com relação ao estado civil 15,4\% (33) eram solteiras, apresentando associação estatística, porém com menor chance de ter vida sexual ativa (Odds Ratio $(O R)=0,18, I C=0,75-0,47, p=<0,001$ ). Referente ao IMC, $62,4 \%$ (139) revelaram sobrepeso e obesidade (IMC de 25 a 30 ou maior que o mesmo), entretanto não obteve significância estatística com relação à interferência na vida sexual e no prazer das mulheres (Tabela 1). 
Tabela 1 - Correlação dos dados sociodemográficos das mulheres atendidas na atenção primária à saúde. Cambé- PR, 2014 - 2016.

\begin{tabular}{|c|c|c|c|c|c|c|}
\hline \multirow{2}{*}{ Variável } & \multirow{2}{*}{$\mathbf{n}$} & \multirow{2}{*}{ (\%) } & \multirow{2}{*}{$\begin{array}{c}\text { Atividade sexual } \\
\text { OR (IC 95\%) }\end{array}$} & \multicolumn{3}{|c|}{ Prazer sexual } \\
\hline & & & & $\mathrm{P}$ & OR (IC 95\%) & $\mathrm{p}$ \\
\hline \multicolumn{7}{|l|}{ Idade } \\
\hline \multirow{2}{*}{$\begin{array}{l}15-24 \text { anos } \\
25-64 \text { anos }\end{array}$} & \multirow{2}{*}{$\begin{array}{l}31 \\
174\end{array}$} & \multirow{2}{*}{$\begin{array}{l}14,4 \\
80,9\end{array}$} & $\begin{array}{c}2,14 \\
(0,40-11,25)\end{array}$ & 0,368 & $\begin{array}{c}1,05 \\
(0,16-6,68)\end{array}$ & 0,954 \\
\hline & & & $\begin{array}{c}2,03 \\
(0,49-8,29)\end{array}$ & 0,325 & $\begin{array}{c}1,62 \\
(0,31-8,53)\end{array}$ & 0,565 \\
\hline 65 anos ou mais & 10 & 4,7 & 1,00 & - & 1,00 & - \\
\hline \multicolumn{7}{|l|}{ Profissão } \\
\hline Sem ocupação & 92 & 43,4 & $\begin{array}{c}1,22 \\
(0,59-2,51)\end{array}$ & 0,577 & $\begin{array}{c}0,578 \\
(0,270-1,239)\end{array}$ & 0,159 \\
\hline Com ocupação & 123 & 56,6 & 1,00 & - & 1,00 & - \\
\hline \multicolumn{7}{|l|}{ Estado civil } \\
\hline Solteira & 33 & 15,4 & $\begin{array}{c}0,18 \\
(0,75-0,47)\end{array}$ & $<0,001^{*}$ & $\begin{array}{c}0,55 \\
(0,19-1,54)\end{array}$ & 0,254 \\
\hline Viúva/ separada & 27 & 12,6 & $\begin{array}{c}0,087 \\
(0,03-0,22)\end{array}$ & $<0,001^{*}$ & $\begin{array}{c}0,96 \\
(0,25-3,59)\end{array}$ & 0,948 \\
\hline Casada/união estável & 155 & 72,0 & 1,00 & - & 1,00 & - \\
\hline \multicolumn{7}{|l|}{ IMC } \\
\hline Sobrepeso e obesos & 139 & 62,4 & $\begin{array}{c}0,75 \\
(0,32-1,76)\end{array}$ & 0,520 & $\begin{array}{c}0,63 \\
(0,26-1,55)\end{array}$ & 0,329 \\
\hline Baixo peso & 11 & 5,4 & $\begin{array}{c}0,28 \\
(0,06-1,18)\end{array}$ & 0,084 & $\begin{array}{c}1,00 \\
(0,10-9,44)\end{array}$ & 1,000 \\
\hline Eutrófico & 65 & 32,2 & 1,00 & - & 1,00 & - \\
\hline Total & 215 & 100,0 & & & & \\
\hline
\end{tabular}

Tabela 2 - Correlação da qualidade de vida das mulheres atendidas na atenção primária à saúde. CambéPR, $2014-2016$.

\begin{tabular}{|c|c|c|c|c|c|c|}
\hline \multirow{2}{*}{ Variável } & \multirow{2}{*}{\multicolumn{2}{|c|}{ n (\%) }} & \multirow{2}{*}{$\begin{array}{c}\text { Atividade sexual } \\
\text { OR (IC 95\%) }\end{array}$} & \multicolumn{3}{|c|}{ Prazer sexual } \\
\hline & & & & $P$ & OR (IC 95\%) & $p$ \\
\hline \multicolumn{7}{|l|}{ Atividade física } \\
\hline Sim & 85 & 39,9 & \multirow{2}{*}{$\begin{array}{c}1,02 \\
(0,49-2,08) \\
1,00 \\
\end{array}$} & 0,963 & \multirow{2}{*}{$\begin{array}{c}0,86 \\
(0,40-1,87) \\
1,00 \\
\end{array}$} & 0,720 \\
\hline Não & 130 & 60,1 & & - & & - \\
\hline \multicolumn{7}{|l|}{ Repouso } \\
\hline Alterado & 33 & 16,3 & \multirow{3}{*}{$\begin{array}{c}0,40 \\
(0,17-0,95) \\
1,00 \\
\end{array}$} & $0,038^{*}$ & \multirow{3}{*}{$\begin{array}{c}0,63 \\
(0,23-1,74) \\
1,00 \\
\end{array}$} & \multirow{2}{*}{$\begin{array}{c}0,378 \\
-\end{array}$} \\
\hline Não alterado & 182 & 83,7 & & - & & \\
\hline \multicolumn{5}{|l|}{ Trabalho doméstico } & & \\
\hline Não recebe ajuda & 142 & 64,7 & \multirow{2}{*}{$\begin{array}{c}0,85 \\
(0,40-1,82) \\
1,00 \\
\end{array}$} & 0,687 & \multirow{2}{*}{$\begin{array}{c}1,10 \\
(0,50-2,44) \\
1,00 \\
\end{array}$} & \multirow{2}{*}{$\begin{array}{c}0,801 \\
- \\
\end{array}$} \\
\hline Recebe ajuda & 73 & 35,3 & & - & & \\
\hline \multicolumn{7}{|l|}{ Dores musculares } \\
\hline Sim & 162 & 73,1 & \multirow{2}{*}{$\begin{array}{c}1,55 \\
(0,70-3,40) \\
1,00\end{array}$} & 0,272 & \multirow{2}{*}{$\begin{array}{c}1,07 \\
(0,43-2,62) \\
1,00\end{array}$} & \multirow{2}{*}{$\begin{array}{c}0,883 \\
-\end{array}$} \\
\hline Não & 53 & 26,9 & & - & & \\
\hline Total & 215 & 100,0 & & & & \\
\hline
\end{tabular}


Quanto a prática de atividade física, mais da metade 60,1\% (130) não a praticavam regularmente. Relacionado aos hábitos de sono, aquelas que referiram problemas para dormir 16,3\% (33) tiveram menor chance de terem atividade sexual $(\mathrm{OR}=0,40, \mathrm{IC} 95 \%=0,17-0,95, \mathrm{p}=0,038)$. Com relação às atividades domésticas, grande parte das mulheres referiram fazer suas atividades diárias sem auxílio de outra pessoa $(64,7 \%, n=142)$, sendo que $73,1 \%$ (162) referiram que sentiam dores musculares. Porém, não houve associação para o presente estudo (Tabela 2).

Dentre as mulheres avaliadas $46 \%$ (99) tiveram de uma a duas gestações, 62,9\% (137) tiveram parto normal, 26,2\% (55) tiveram pelos menos um aborto. Quando nos referimos aos métodos contraceptivos 45,1\% (108) não utilizam métodos para prevenção de infecções sexualmente transmissíveis ou gravidez (Tabela 3).

O método hormonal $27,7 \%$ (54) foi o mais utilizado pelas mulheres avaliadas e demonstrou que há significância estatística e uma maior chance do seu uso nessa população, quando relacionado com atividade sexual (OR=4,71, IC 95\% 1,69-13,12 e $p=0,003$ ). O mesmo ocorreu com relação ao prazer sexual, demonstrado que mesmo utilizando método hormonal essas mulheres apresentaram maior chance de terem prazer na relação sexual (OR=3,90 e IC 95\%=1,34-1,35 e p=0,012) (Tabela 3).

Tabela 3 - Correlação dos dados ginecológicos e obstétricos das mulheres atendidas na atenção primária à saúde. Cambé- PR, 2014 - 2016.

\begin{tabular}{|c|c|c|c|c|c|c|}
\hline \multirow{2}{*}{ Variável } & \multirow{2}{*}{$\mathbf{n}$} & \multirow{2}{*}{ (\%) } & \multirow{2}{*}{$\begin{array}{c}\text { Atividade sexual } \\
\text { OR (IC 95\%) }\end{array}$} & \multicolumn{3}{|c|}{ Prazer sexual } \\
\hline & & & & $\mathrm{P}$ & OR (IC 95\%) & $\mathrm{p}$ \\
\hline \multicolumn{7}{|l|}{ Número de Gestações } \\
\hline Nenhuma & 29 & 13,5 & \multirow{3}{*}{$\begin{array}{c}1,12 \\
(0,39-3,15) \\
1,93 \\
(0,89-4,20) \\
1,00\end{array}$} & 0,829 & \multirow{2}{*}{$\begin{array}{c}3,23 \\
(0,68-15,29) \\
1,51 \\
(0,69-3,34)\end{array}$} & \multirow{2}{*}{$\begin{array}{l}0,139 \\
0,300\end{array}$} \\
\hline Uma a duas gestações & 99 & 46,0 & & 0,096 & & \\
\hline Três ou mais gestações & 87 & 40,5 & & - & 1,00 & - \\
\hline \multicolumn{7}{|l|}{ Parto cesárea } \\
\hline Sim & 109 & 50,5 & \multirow{2}{*}{$\begin{array}{c}1,17 \\
(0,58-2,38) \\
1,00\end{array}$} & 0,6477 & \multirow{2}{*}{$\begin{array}{c}1,84 \\
(0,85-3,99) \\
1,00\end{array}$} & 0,120 \\
\hline Não & 106 & 49,5 & & - & & - \\
\hline \multicolumn{7}{|l|}{ Parto normal } \\
\hline Sim & 137 & 62,9 & \multirow{2}{*}{$\begin{array}{c}0,72 \\
(0,34-1,52) \\
1,00\end{array}$} & 0,392 & \multirow{2}{*}{$\begin{array}{c}0,56 \\
(0,24-1,30) \\
1,00\end{array}$} & \multirow{2}{*}{$\begin{array}{c}0,182 \\
-\end{array}$} \\
\hline Não & 78 & 37,1 & & - & & \\
\hline \multicolumn{7}{|l|}{ Aborto } \\
\hline Sim & 55 & 26,2 & \multirow{2}{*}{$\begin{array}{c}0,83 \\
(0,38-1,83) \\
1,00\end{array}$} & 0,657 & \multirow{2}{*}{$\begin{array}{c}0,62 \\
(0,27-1,41) \\
1,00\end{array}$} & \multirow{2}{*}{$\begin{array}{c}0,259 \\
-\end{array}$} \\
\hline Não & 160 & 73,8 & & - & & \\
\hline \multicolumn{7}{|l|}{ Método Contraceptivo } \\
\hline Barreira & 25 & 12,8 & \multirow{4}{*}{$\begin{array}{c}11,30 \\
(1,45-87,89) \\
4,71 \\
(1,69-13,12) \\
6,39 \\
(1,41-28,79) \\
1,00\end{array}$} & $0,020^{*}$ & \multirow{3}{*}{$\begin{array}{c}10,00 \\
(1,25-79,45) \\
3,90 \\
(1,34-1,35) \\
2,09 \\
(0,69-6,29)\end{array}$} & $0,029^{*}$ \\
\hline Hormonal & 54 & 27,7 & & $0,003^{*}$ & & $0,012^{*}$ \\
\hline Cirúrgico & 28 & 14,4 & & $0,016^{*}$ & & 0,190 \\
\hline Não utiliza & 108 & 45,1 & & - & 1,00 & - \\
\hline Total & 215 & 100,0 & & & & \\
\hline
\end{tabular}

Legenda: Odds Ratio (OR), $p<0,05 \%$ e Intervalo de confiança 95\% (IC 95\%). Fonte: Malta NJF, Cestari MEW, Pisicchio RJ, et al., 2019.

Em segundo lugar, o método cirúrgico foi o mais referido pelas mulheres com 14,4\% (28). Este apontou associação estatística com atividade sexual $(\mathrm{OR}=6,39, \mathrm{IC}=1,41-28,79$ e $\mathrm{p}=0,016)$, seguida da utilização de métodos de barreira 12,8\% (25), apresentando significância estatística com relação atividade sexual 
$(\mathrm{OR}=11,30, \mathrm{IC}=1,45-87,89$ e $\mathrm{p}=0,020)$ apresentando maior chance de uso quando comparado aos demais métodos e o mesmo ocorreu no prazer sexual ( $O R=10,00, I C=1,25-79,45$ e $p=0,029)$ (Tabela 3).

Tratando-se da atividade sexual, $82,17 \%$ (177) apresentavam vida sexual ativa e $81,7 \%$ (182) referiam sentir prazer sexual, sendo que $71,2 \%$ (156) não apresentavam queixas sexuais. Entretanto, essas mulheres que apresentaram dispareunia e sangramento $28,8 \%$ (59) e tinham atividade sexual $(O R=3,97, I C 95 \%=1,13$ $11,79, p=0,013)$ todavia, apresentavam uma chance menor de terem prazer sexual durante $o$ ato $(O R=0,21$, IC $95 \%=0,09-0,49, p<0,001)$. Mais da metade das mulheres referiram não estar satisfeitas com o corpo $54,2 \%$ (122) e 109 (89\%) estavam se sentindo acima do peso e com a sua imagem corporal alterada (Tabela 4).

Tabela 4 - Correlação dos dados sobre prazer, atividade sexual e satisfação corporal das mulheres atendidas na atenção primária à saúde. Cambé-PR, 2014-2016.

\begin{tabular}{|c|c|c|c|c|c|c|}
\hline \multirow{2}{*}{ Variável } & \multirow{2}{*}{$\mathbf{n}$} & \multirow{2}{*}{ (\%) } & \multicolumn{2}{|c|}{ Atividade sexual } & \multicolumn{2}{|c|}{ Prazer sexual } \\
\hline & & & OR (IC 95\%) & $\mathrm{P}$ & OR (IC 95\%) & $p$ \\
\hline \multicolumn{7}{|l|}{ Atividade sexual } \\
\hline Sim & 177 & 82,1 & - & - & $\begin{array}{c}1,98 \\
(0,48-8,12)\end{array}$ & 0,340 \\
\hline Não & 38 & 17,9 & - & - & 1,00 & - \\
\hline \multicolumn{7}{|c|}{ Prazer na relação sexual } \\
\hline Sim & 182 & 81,7 & $\begin{array}{c}1,98 \\
(0,48-8,12)\end{array}$ & 0,340 & - & - \\
\hline Não & 33 & 18,3 & 1,00 & - & - & - \\
\hline \multicolumn{7}{|l|}{ Queixa sexual } \\
\hline $\begin{array}{l}\text { Dispareunia ou } \\
\text { sangramento }\end{array}$ & 59 & 28,8 & $\begin{array}{c}3,97 \\
(1,13-11,79) \\
1.00\end{array}$ & $0,013^{*}$ & $\begin{array}{c}0,21 \\
(0,09-0,49) \\
1.00\end{array}$ & $<0,001^{*}$ \\
\hline Sem queixas & 156 & 71,2 & & - & & - \\
\hline \multicolumn{7}{|l|}{ Satisfação corporal } \\
\hline Sim & 93 & 45,8 & $\begin{array}{c}1,17 \\
(0,55-2,50)\end{array}$ & 0,673 & $\begin{array}{c}0,47 \\
(0,22-1,03)\end{array}$ & 0,061 \\
\hline Não & 122 & 54,2 & 1,00 & - & 1,00 & - \\
\hline \multicolumn{7}{|c|}{$\begin{array}{l}\text { Motivo para insatisfação } \\
\text { corporal }\end{array}$} \\
\hline $\begin{array}{l}\text { Sobrepeso e gordura } \\
\text { abdominal }\end{array}$ & 109 & 89,0 & $\begin{array}{c}1,02 \\
(0,45-2,29)\end{array}$ & 0,950 & $\begin{array}{c}2,00 \\
(0,90-4,42)\end{array}$ & 0,087 \\
\hline Outros & 13 & 11,0 & $\begin{array}{c}0,40 \\
(0,11-1,51)\end{array}$ & 0,181 & $\begin{array}{c}3,00 \\
(0,35-25,17)\end{array}$ & 0,311 \\
\hline Está satisfeita & 92 & 46,2 & 1,00 & - & 1,00 & - \\
\hline
\end{tabular}

Legenda: Odds Ratio (OR), p <0,05\% e Intervalo de confiança 95\% (IC 95\%).

Fonte: Malta NJF, Cestari MEW, Pisicchio RJ, et al., 2019.

\section{DISCUSSÃO}

Quanto aos fatores que interferem no prazer e na atividade sexual das mulheres e a relação destes com o perfil sociodemográfico, ginecológico e obstétrico, observou-se que não houve associação significativa entre as variáveis dependentes e o índice de massa corporal, entretanto, resultados discordantes são encontrados na literatura, mostrando que o sobrepeso e obesidade podem interferir significativamente na sexualidade do indivíduo (SILVA BM, et al., 2012). Essa interferência ocorre especialmente devido a alteração na função vascular, endotelial e hormonal (SILVA BM, et al., 2012). Em especial, o fluxo sanguíneo genital secundário e a aterosclerose da artéria hipogástrica, além de fatores hormonais e psicogênicos favorecem a síndrome vascular vaginal e clitoriana, sendo uma importante causa de disfunção sexual (JORGE LB, et al., 2015). 
Segundo Mariano MLL, et al. (2014), para que aconteça uma vida sexual saudável e satisfatória, deve ocorrer o aumento da autoestima por meio da auto aceitação, facilitando a aceitação de si mesmo com o outro ser. Neste sentido, a reconstrução da imagem corporal, o bem-estar físico e psicológico pode ocasionar em maior disposição física e emocional, além do resgate do convívio social, aumento da vaidade, desejo, sensualidade e segurança frente ao parceiro na vivência da sua sexualidade. No presente estudo o sobrepeso e obesidade não interferiu na vida sexual e prazer das mulheres, porém acredita-se que os profissionais de saúde devem estimular as mulheres quanto ao autocuidado para manutenção do peso adequado, favorecendo prevenção de doença cardiovascular e melhora do autoestima, contribuindo para a qualidade da vida sexual (SILVA BM, et al.,2012).

Quanto ao uso de anticoncepcional hormonal, houve relação com a atividade sexual e prazer sexual. O método hormonal proporciona às mulheres liberdade de escolha com segurança sobre o melhor momento de engravidar, proporcionando liberdade sexual sem o medo de uma gravidez não planejada, além de ser um dos métodos fornecidos pelo Sistema Único de Saúde, de relativo fácil acesso e utilização (LOPES GP, et al., 2008). Em um estudo clínico randomizado, que investigou o efeito dos contraceptivos orais combinados sobre a sexualidade feminina, identificou que seu uso à base de estradiol, está associado a redução mínima do interesse sexual, sugerindo não ter uma relevância clínica o seu uso para o desejo sexual (LUNDIN C, et al., 2018).

A utilização do método contraceptivo cirúrgico teve associação com a realização de atividade sexual, demonstrando que estas mulheres, têm mais chance de ter atividade sexual do que as que utilizam método contraceptivos hormonais. Ao considerar-se esse procedimento permanente e de alta eficácia para prevenção da gravidez, é provável que possa proporcionar uma sensação de liberdade sexual. Soma-se a isso, a evidência de que a maioria das mulheres realizam este procedimento na maturidade da sua sexualidade, o que também poderia explicar a significância relacionada a atividade sexual. Essa é uma questão que apresenta uma lacuna de evidências a serem exploradas em novos estudos (GONÇALVES GHT e MARCON SS, 2006).

Referente ao método de barreira, houve associação significativa com relação ao prazer e atividade sexual, demonstrando que há mais chance do seu uso, do que os demais métodos. O preservativo masculino é o terceiro método mais utilizado pela população estudada, entretanto, resultados divergentes são encontrados na literatura demonstrando que a sua utilização muitas vezes não é uma das primeiras escolhas, consideradas desconfortáveis, limitando a sensibilidade, ereção e prazer (FRANCISCO MTR, et al., 2016). O Ministério da Saúde (2008), afirma que o preservativo é um método seguro e protege contra as infecções sexualmente transmissíveis. Ressalta-se que o uso do método de barreira além de ter o objetivo de prevenir doenças sexualmente transmissível, proporciona ao casal a liberdade de não depender de um planejamento prévio e/ou risco de esquecer de tomar o contraceptivo. Neste sentido, o uso do preservativo deve ser estimulado por profissionais de saúde por não apresentar custo ao casal, assim como a facilidade de acesso nas Unidades de Atenção Primária a Saúde.

Outra categoria explorada foi sobre sono e repouso das mulheres. No presente estudo observou-se significância estatística com relação a atividade sexual, demonstrando que quando as mulheres apresentam problemas com o sono, a chance de terem atividade sexual é baixa. A perturbação do sono pode levar a disfunção do funcionamento físico, ocupacional, cognitivo e social do indivíduo, comprometendo sua qualidade de vida (MULLER MR e GUIMARÃES SS, 2007). Diante disso podemos observar que a atividade sexual também é afetada quando a qualidade do sono é perturbada. Um estudo que avaliou a qualidade do sono, identificou que o prazer sexual teve relação com desfecho favorável para o sono. Os autores identificaram ainda que quando o prazer é alcançado por meio da auto estimulação, há uma melhor qualidade de sono, sugerindo que a atividade sexual com orgasmo favorece melhor qualidade no sono (LASTELLA M, et al., 2019).

O profissional de saúde deve compreender a mulher dentro do seu contexto social, físico e emocional, buscando os motivos que fazem com que esta mulher não esteja alcançando uma qualidade no sono, proporcionando apoio para sua resolutividade, reconhecendo que o prazer sexual pode ser uma estratégia 
para melhorias da qualidade do sono (ROBAINA JR, et al., 2015). A idade média das mulheres pesquisadas estava compreendida dentro da fase do climatério, entretanto a variável idade não apresentou significância estatística. Porém, sabe-se que as mulheres que estão dentro desta faixa etária podem apresentar maiores dificuldades na sexualidade, principalmente devido às alterações psicológicas e hormonais que podem influenciar no seu bem-estar e no padrão de sexualidade (ALVEZ ERP, et al., 2015).

Grande parte das mulheres pesquisadas no presente estudo não tiveram queixas sobre sua sexualidade. Entretanto, houve chance de apresentarem dispareunia e ou sangramento nas relações sexuais. $O$ prazer sexual se mostrou significativo, demonstrando que durante uma relação sexual com dor o prazer tende a diminuir. Neste contexto, a consulta de coleta de citologia oncótica pode ser uma estratégia na prevenção e tratamento de doenças ginecológicas, sendo também uma excelente oportunidade para o profissional ouvir a mulher quanto a queixas e dificuldades sexuais, intervindo dentro da sua necessidade específica (FERREIRA MLSM, 2009). Um estudo que avaliou a presença da atividade sexual em mulheres jovens, identificou que entre as mulheres pesquisadas, houve preocupação em satisfazer sexualmente o seu parceiro, negando frequentemente a sua própria satisfação, com o objetivo de manter o relacionamento (FLEURY HJ e ABDO $\mathrm{CHN}, 2009$ ). Neste sentido as mulheres devem ser empoderadas quanto ao poder de escolha, alcançando 0 que desejam, sendo responsáveis por sua própria vida. O empoderamento não pode ser ofertado e ou realizado para pessoas ou grupos, mas sim deve realizar-se em processos em que esses se empoderam de si mesmos. Para que aconteça o empoderamento deve ocorrer uma mudança na dominação tradicional dos homens sobre as mulheres, com garantia de autonomia quanto ao controle do seu corpo, à sua sexualidade e ao seu direito de ir e vir (LANDERDAHL MC, et al., 2013). Esta é uma temática que deve ser mais bem explorada, tendo em vista que as pesquisas ainda são incipientes quanto ao entendimento deste fenômeno.

A disfunção sexual tem sido cada vez mais frequente nas mulheres. Um estudo realizado no município de Cajazeiras- PB com 330 mulheres demonstrou que 50\% apresentavam faixa etária entre 35 a 45 anos sendo que $19,5 \%$ apresentaram dispareunia como a queixa mais frequente seguida da lubrificação diminuída e $73 \%$ apresentavam vida sexual ativa.23 As disfunções sexuais têm ocorrido também nas mulheres mais novas, sendo caracterizada pela falta de desejo sexual e a ausência do orgasmo que tende a aumentar com o passar do tempo (KOBAYASHI C e REIS AS, 2015). Quanto à imagem corporal evidenciou-se insatisfação com seu próprio corpo, porém não houve significância estatística com prazer e atividade sexual. Apesar deste ser um dado subjetivo, pode-se inferir que estas mulheres querem buscar o seu prazer e ter uma atividade harmoniosa com seus parceiros. A insatisfação sexual geralmente vem conjugada pelo medo, falta de desejo, sensação de culpa diante das alterações que ocorrem com o ambiente familiar, principalmente, com o seu corpo e no relacionamento com o parceiro (ALVEZ ERP, et al., 2015).

Identificou-se que grande parte das mulheres realizavam as tarefas domésticas sozinhas, sem ajuda de familiares ou outras pessoas. Apesar das análises não serem significativas, podemos observar que na literatura os conflitos conjugais se iniciam com a dificuldade da mulher em conciliar as responsabilidades assumidas nos tempos modernos, caracterizados pela dupla jornada de trabalho, interferindo na relação conjugal e consequentemente, na vida sexual do casal (OZORIO CD, 2016). As mulheres têm conquistado espaço no mundo profissional, ocupando cargos já considerados de exclusividade masculina. Porém, ainda não abdicaram das responsabilidades relacionadas ao lar. Por esse motivo, um relacionamento de complementaridade, relacionando de forma igualitária com funções distribuídas entre os cônjuges, demonstra carinho, respeito, tolerância, expressão de sentimentos, compreensão e diálogo entre o casal (OZORIO CD, 2016).

\section{CONCLUSÃO}

Os resultados desse estudo evidenciam que a saúde reprodutiva da mulher deve ser valorizada e observada de forma holística durante as consultas de enfermagem. A inclusão desta temática pode favorecer o empoderamento e permitir a construção de um desenvolvimento sexual pautada na complementariedade e horizontalidade. Como limitação do presente estudo destaca-se a abordagem quantitativa, sugerindo que pesquisas qualitativas possam significar o fenômeno, porém o presente artigo tem dados representativos, servindo de base para novas pesquisas. 


\section{REFERÊNCIAS}

1. ALMEIDA AAB, et al. Influências do climatério na atividade sexual feminina. Rev. Rene, 2016; 3(17):422-426.

2. ALVES ERP, et al. Climatério: a intensidade dos sintomas e o desempenho sexual. Texto Contexto Enferm. 2015; 24(1):64-71.

3. BARBOSA HHG, ALMEIDA JUNIOR VA. (Des) Igualdade de gênero: restrições à autonomia da mulher. Pensar, 2017; 22(1):240-271.

4. BEAUVOIR SO. Segundo sexo: A experiência vivida. Ed. Nova Fronteira: Rio de Janeiro; 1980.

5. FERREIRA MLSM. Motivos que influenciam a não-realização do exame de papanicolaou segundo a percepção de mulheres. Esc. Anna Nery Rev Enferm, 2009; 13(2): 378-384.

6. FLEURY HJ, ABDO CHN. Desejo sexual feminino. Diagn Tratamento, 2009; 14(1):47-51.

7. FRANCISCO MTR, et al. Condom use among participants of the Carnival - gender perspective. Esc. Anna Nery, 2016; 1(20):106-113.

8. GONÇALVES GHT, MARCON SS. Laqueadura ou vasectomia: fatores a considerar para a saúde da mulher. Ciênc. Biol. Saúde, 2006; 8(1):31-38.

9. Instituto Brasileiro de Geografia e Estatística (IBGE). População no último censo de Cambé-Pr. 2015; disponível em: http://cidades.ibge.gov.br/brasil/pr/cambe/panorama

10. JORGE LB, et al. Impacto do sobrepeso na disfunção sexual feminino: revisão da literatura. Feminina, 2015; 43(6):265- 271.

11. KOBAYASHI C, REIS AS. Início da atividade sexual de mulheres jovens: questionando sua satisfação e preferências. Bol. Psicol., 2015; 65(143):123-130.

12. LANDERDAHL MC, et al. Processo de empoderamento feminino mediado pela qualificação para o trabalho na construção civil. Esc. Anna Nery, 2013; 17(2):306-312.

13. LASTELLA M, et al. Sex and Sleep: Perceptions of Sex as a Sleep Promoting Behavior in the General Adult Population. Front Public Health, 2019; 7(33).

14. LEVY L, GOMES IC. Relação conjugal, violência psicológica complementaridade fusional. Psic. Clin., 2008; 20(2):163-172.

15. LOPES GP, AMBROGINI CC, MEGALE A. Contracepção hormonal e sexualidade. Revista Brasileira de Medicina, 2008; 65(2):3-11.

16. LUNDIN C, et al. Sexual function and combined oral contraceptives - a randomised, placebo-controlled trial. Endocr Connect, 2018; 7(11):1208-1216.

17. MARIANO MLL, et al. Bariatric surgery: impact on sexuality of the obese person. Rev. Col. Bras. Cir., 2014; 41(6):412420.

18. MENEZES LO, LIMA FAM. O erotismo nos quadrinhos: ferramenta artística, política e social. Entre palavras, 2015; 5(5):269-278.

19. Ministério da saúde (BR). Datasus. Informações completas sobre o município de Cambé-Pr. Brasília; 2010.

20. Ministério da Saúde (BR). Secretaria de Atenção à Saúde. Departamento de Atenção Básica. Saúde sexual e saúde $\begin{array}{llll}\text { reprodutiva. } & \text { Brasília; } 2013 . & \text { Disponível }\end{array}$ http://bvsms.saude.gov.br/bvs/publicacoes/saude_sexual_saude_reprodutiva.pdf

21. MULLER MR, GUIMARÃES SS. Impacto dos transtornos do sono sobre o funcionamento diário e qualidade de vida. Estud. Psicol., 2007; 4(4):519-528.

22. OZORIO CD. Trabalho e família: repercussões das transformações sociais nas relações humanas. Dignidade Revista, 2016; 1(2):11-20.

23. ROBAINA JR, et al. Fatores psicossociais e socioeconômicos relacionados à insônia e menopausa: estudo pró-saúde. Cad. Saúde Pública, 2015; 31(3): 597-606.

24. SCHLOSSER A, CAMARGO BV. Contribuições de pesquisas brasileiras sobre o amor e relacionamentos amorosos. Temas psicol., 2014; 22(4):795-808.

25. SILVA BM, et al. Cavalcante JC. Incidence of sexual dysfunction in patients with obesity and overweight. Rev. Col. Bras. Cir., 2012; 40(3):196-202.

26. SOUZA NS, BERTGES LR, PEREIRA VC. Maria Teresa Horta: Escrita feminina na poesia de um corpo liberto. Interdisciplinar, 2016; 26(1):123-136. 